\title{
Reduction of background in CR-39 SSNTD using chemical pre-etching methods
}

\author{
Rupali Rohatgi*, Deepa Sathian, V Jayalakshmi, Sarala Nair, P K Marathe, \\ G Chaurasiya and S Kannan \\ Personnel Monitoring Section, RP \& AD, Bhabha Atomic Research Centre, Mumbai-400 094, India \\ E-mail : rupalirohatgi@rediffmail.com
}

\begin{abstract}
Presently, CR-39 Solid State Nuclear Track Detectors (SSNTD) is being used for Fast Neutron Personnel Monitoring in India. Individual detectors are cut manually from CR-39 sheets. Laser-cutting of the sheets will ensure precision and also save on the laborious efforts involved in sheet cutting. It was observed that there is a slight increase in background tracks due to laser-cutting. Studies were carried out on the effect of chemical pre-etching at higher temperatures in properties of CR-39 such as the background, sensitivity and minimum measurable dose. For this purpose a pre-etch step was included in the normal processing of the CR-39 foils in an elevated temperature electrochemical cell (ETECE cell) and the duration for pre-etch step at elevated temperature was optimized.
\end{abstract}

Samples of CR-39 exposed to dose equivalents ranging from $1 \mathrm{mSv}$ to $10 \mathrm{mSv}$ using Am-Be neutron source were processed by both the methods, under normal processing using ECE cell and in the optimized chemical pre-etch followed by normal processing. The dose responses of CR-39 processed by both methods are compared in this paper.

Keywords : CR-39, SSNTD, fast neutron monitoring, chemical pre-etching, sensitivity.

PACS Nos. : $29.40 .-n, 87.53 . \mathrm{Bn}, 81.65 . \mathrm{Cf}$

\section{Introduction}

CR-39 Solid State Nuclear Track Detectors (SSNTD), is a wide ranged proton sensitive organic polymer insensitive to beta and gamma radiation doses $[1,2]$. There is no post irradiation fading of damaged tracks due to environmental conditions in CR-39 and it offers a better energy response with a threshold at $100 \mathrm{keV}$. Properties of CR-39 make it a versatile neutron detector as neutrons are always accompanied by gamma radiation. However, there is a considerable increase of the inherent background and decrease in sensitivity of CR-39 with time (aging). CR-39 sheets are being stored in black polythene in refrigerator to control aging. Presently, CR-39 imported from Pershore Moulding, U.K. is being used for nationwide fast neutron personnel monitoring in India. 
CR-39 detectors of dimension $3 \mathrm{~cm} \times 3 \mathrm{~cm}$, are cut manually from sheets of dimension $20 \mathrm{~cm} \times 25 \mathrm{~cm}$ and sealed in triple laminated pouches with $1 \mathrm{~mm}$ thick polyethylene radiator of same dimension in front of it for enhanced sensitivity, and used as a personnel monitor. It is now an international practice, to cut the sheets with Carbon Dioxide $\left(\mathrm{CO}_{2}\right)$ laser beam. This paper is aimed at studying the changes if any in the background or sensitivity in CR-39 foils due to the use of laser beam for cutting and also exploring methods to reduce the background tracks in the CR-39 SSNTD using chemical pre-etch methods.

\section{Materials and methods}

CR-39 foils were cut, both manually as well as by $\mathrm{CO}_{2}$ laser beam for use in this study. A set of 40 foils of each type with radiator in front, are exposed in air to 1 $\mathrm{mSv}$ from a $1 \mathrm{Ci} \mathrm{Am}-\mathrm{Be}$ neutron source, and another set of 40 foils served as a control. The irradiations were performed in a laboratory under low scatter conditions, at a distance of $50 \mathrm{~cm}$ in air, with the radiator side facing the $1 \mathrm{Ci} \mathrm{Am-Be}$ neutron source having a neutron yield of $2.5 \times 10^{6} \mathrm{n} / \mathrm{sec}$. Four exposed foils along with equal number of control foils of each type were processed by (i) normal etching condition used for personnel monitoring and (ii) different chemical pre-etch durations of $6,7,8$ and 9 hours, followed by normal electro chemical etching in an ETECE cell at $60^{\circ} \mathrm{C}$. The tracks so produced are counted using a PC based image analysis system. The results are tabulated in Tables 1 and 2 .

Table 1. Background and sensitivity of Laser cut and manual cut CR-39 foils.

\begin{tabular}{lcccccc}
\hline & $\begin{array}{c}\text { Avg. } \\
\text { Track } \\
\text { dia. in } \\
\mu \mathrm{m}\end{array}$ & $\begin{array}{c}\text { Control } \\
\text { Avg. } \\
\text { tracks } \\
\mathrm{cm}^{-2}\end{array}$ & $\begin{array}{c}\text { Avg. } \\
\text { Tracks } \\
\mathrm{cm}^{-2} \text { for } \\
1 \mathrm{mSv}\end{array}$ & $\begin{array}{c}\text { Net. } \\
\text { Tracks } \\
\mathrm{cm}^{-2} \\
\mathrm{mSv}^{-1}\end{array}$ & $\begin{array}{c}\mathrm{S} / \mathrm{N} \\
\text { ratio }\end{array}$ & $\begin{array}{c}\mathrm{MDL} \\
(\mathrm{mSv})\end{array}$ \\
\hline Manual cut & 95.7 & $15 \pm 3$ & 110 & $95 \pm 4$ & 7.3 & 0.12 \\
Laser cut & 100 & $22 . \pm 9$ & 108 & $86 \pm 24$ & 4.7 & 0.30 \\
\hline
\end{tabular}

\subsection{Normal processing conditions for CR-39 in personnel monitoring :}

The foils used for personnel monitoring are normally processed by Elevated Temperature Electro Chemical Etching (ETECE) [3]. The processing was carried out in two stages using a specially designed Electro Chemical Etching Cell (ECE cell), which processes 16 foils at a time. At one side of the cell a plain steel plate is kept to serve as neutral electrode, while at the other side small cavities filled with $7 \mathrm{~N} \mathrm{KOH}$ solution contains an electrode dipped into the $\mathrm{KOH}$ solution through which current is allowed to pass by applying high voltage. The foils are mounted on the ECE cell such that the side of the foil, which has been exposed to radiation through the $1 \mathrm{~mm}$ thick polyethylene radiator, faces the electrode dipped in $\mathrm{KOH}$ solution. The etching of the foil is carried out in a incubator maintained at $60^{\circ} \mathrm{C}$ in two steps. For the first step - etching at low 
Table 2. Effect of changes in duration of pre-etched on background and sensitivity of CR-39 foils with values in bracket for laser cut foils.

\begin{tabular}{llllll}
\hline Etching condition & $\begin{array}{l}\text { Avg. track dia. } \\
\mu \mathrm{m}\end{array}$ & $\begin{array}{l}\text { Control (Avg.) } \\
\text { tracks } \mathrm{cm}^{-2}\end{array}$ & $\begin{array}{l}\text { (Avg.) tracks } \\
\mathrm{cm}^{-2} \text { for } 1 \mathrm{mSv}^{2}\end{array}$ & $\begin{array}{l}\text { Net. tracks } \mathrm{cm}^{-2} \\
\mathrm{msv}^{-1}\end{array}$ & $\mathrm{~S} / \mathrm{N}$ ratio \\
\hline Normal ETECE & 108 & $15 \pm 2.98$ & 110 & $95 \pm 3.77$ & 7.33 \\
& $(99.6)$ & $(22 \pm 9.0)$ & $(108.6)$ & $(86 \pm 24.5)$ & $(4.8)$ \\
$1 \mathrm{hr}$ pre-etch + & 97.5 & $6.75 \pm 1.9$ & 83.5 & $76.75 \pm 15.7$ & 12.8 \\
ETECE & $(85)$ & $(23 \pm 7.87)$ & $(97.5)$ & $(74.5 \pm 18.6)$ & $(4.2)$ \\
$11 / 4 \mathrm{hr}$ pre-etch + & 103.66 & $11 \pm 1.73$ & 84 & $73 \pm 18.52$ & 7.6 \\
ETECE & $(102.6)$ & $(8.6 \pm 5.0)$ & $(99.6)$ & $(91.0 \pm 11.5)$ & $(11.5)$ \\
$11 / 2 \mathrm{hr}$ pre-etch + & 91.33 & $8.66 \pm 2.1$ & 113.99 & $105.33 \pm 20.9$ & 13.1 \\
ETECE & $(83.66)$ & $(16.0 \pm 3.0)$ & $(112.6)$ & $(96.6 \pm 18.7)$ & $(7.0)$ \\
$2 \mathrm{hr}$ pre-etch + & 91.33 & $10.66 \pm 2.51$ & 139.66 & $129 \pm 21$ & 13.0 \\
ETECE & $(123.6)$ & $(11.3 \pm 6.65)$ & $(107.9)$ & $(96.6 \pm 15)$ & $(9.5)$ \\
$21 / 2 \mathrm{hr}$ pre-etch + & 111 & $9.6 \pm 4.5$ & 215.2 & $205.6 \pm 35.2$ & 22.4 \\
ETECE & $(86)$ & $(53 \pm 19)$ & $(186)$ & $(133 \pm 26)$ & $(3.5)$ \\
\hline
\end{tabular}

frequency, the power supply operates at 1250 volts constant potential and low frequency of $100 \mathrm{~Hz}$ for 4 hours. In this stage the damaged tracks are marked from the back ground distinctly. This is followed by the second step - etching at high frequency of $3.5 \mathrm{kHz}$ at 1250 volts for $40 \mathrm{~min}$ duration. In this step the electrical stresses enlarge the tracks marked during the low frequency operation. The cell with the CR-39 foils is cooled for 2 hrs after the ETECE etch process after which the foils are washed thoroughly and dried at room temperature for eight to ten hrs. Total processing time required was $6 \mathrm{hrs}$ and $40 \mathrm{~min}$ for etching 16 foils. The foils exposed to doses ranging from $1 \mathrm{mSv}$ to $10 \mathrm{mSv}$ were etched under the normal conditions for the dose response curve as shown in Figure 1.

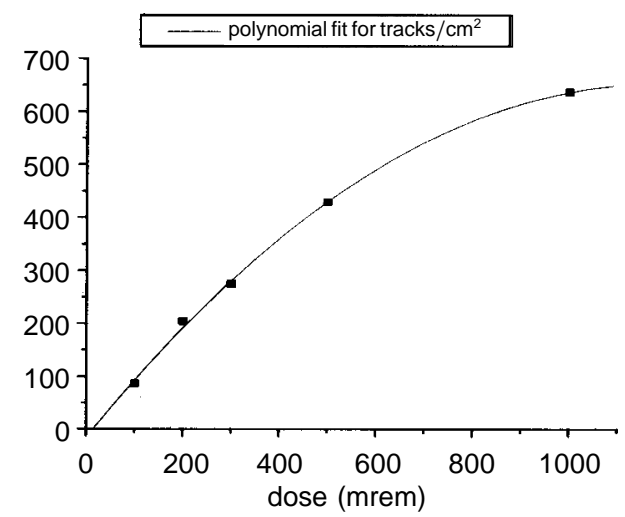

Figure 1. Dose response of CR-39 for normal etching conditions. 


\subsection{Chemical pre-etch method for reduction in background tracks :}

Studies were carried out on the effect of chemical pre-etching [4] at elevated temperatures for durations ranging from $1-3$ hours at $60^{\circ} \mathrm{C}$, in properties of CR-39 such as the background and sensitivity. For this purpose an additional pre-etch step was included in the normal processing of the CR-39 foils using ETECE cell. The foil is mounted with the side exposed to radiation though the $1 \mathrm{~mm}$ thick polyethylene radiator facing the electrode dipped in $\mathrm{KOH}$ solution in the ETECE cell. The etching of the foil is carried out in a incubator maintained at $60^{\circ} \mathrm{C}$ in three steps. For the first step which is the pre-etch step, the ECE cell is placed in the incubator at $60^{\circ} \mathrm{C}$ for duration varying from $1 \mathrm{hr}$, to $3 \mathrm{hrs}$ without applying voltage, followed by the second step - etching at low frequency where the power supply operates at a low frequency of $100 \mathrm{~Hz}$ and 1250 volts constant potential for 3 hours. This is followed by the third step - etching at high frequency of $3.5 \mathrm{kHz}$ for $40 \mathrm{~min}$ duration. The ECE is cooled for 30 min after the ETECE etch process, after which the foils are washed and dried at room temperature for 8 to $10 \mathrm{hrs}$. The duration for pre-etch was optimized based on the background, sensitivity and signal to noise ratio for different pre-etch durations employed. The foils exposed to doses ranging from $1 \mathrm{mSv}$ to $10 \mathrm{mSv}$ were etched under the optimum pre-etch conditions and the dose response curve is presented in Figure 2.

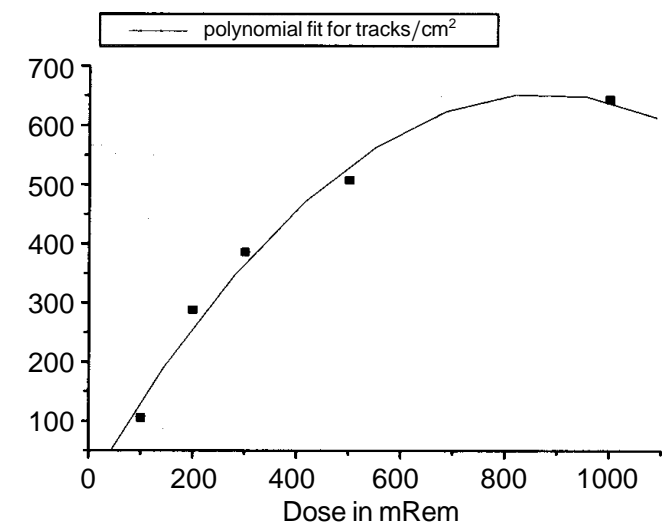

Figure 2. Dose respose of CR-39 for optimised chemical pre-etch condition.

\subsection{Image analysis system used for track counting of CR-39 foils :}

For the counting of tracks developed in the processed foils an image analysis system [5] developed by Electronics Division, BARC is used. This consists of video camera, image frame grabber, and suitable light source all fixed in a light proof box having a small sliding tray on which the processed foil is mounted, and an image analysis software. The control foil (unexposed, processed foil) is first mounted and the track diameter in micron is selected, above which tracks are to be counted. A program has been developed which counts the tracks of size above the selected track diameter over 
the area of $1 \mathrm{sq} \mathrm{cm}$, eliminating all other overlapping tracks or deformities. The no of tracks recorded by the control is stored in the program. The exposed and processed foils are then scanned through the system. The control tracks recorded is subtracted from the tracks of exposed foils to give net tracks, which are multiplied by a calibration factor relevant to the neuron source to arrive at the equivalent dose.

\section{Results}

The background and sensitivity of the foils manually cut are compared with the background and sensitivity of the laser cut foils in Table 1. A $10 \%$ increase in background of laser cut foils in comparison to the manual cut foils is due to the exposure of CR-39 foils to environmental radiation while in the factory.

The response of CR-39 for various pre-etch durations both for hand-cut and lasercut foils have been shown in Table 2. The chemical pre-etch for $90 \mathrm{~min}$ followed by ETECE for $3 \mathrm{hrs}$ at low frequency and $40 \mathrm{~min}$ high frequency seems optimum for the processing and evaluation set up available at personnel monitoring section. The dose response of CR-39 for Am-Be neutron source, for both normal and for optimum pre-etch conditions is presented in Table 3.

Table 3. Dose response of CR-39 Foils.

\begin{tabular}{lccccccccccc}
\hline & \multicolumn{4}{c}{ Normal ecthed } & \multicolumn{4}{c}{ Chemical pre-etched } \\
\hline Neutron dose $(\mathrm{mSv})$ & 1.0 & 2.0 & 3.0 & 5.0 & 10.0 & 1.0 & 2.0 & 3.0 & 5.0 & 10.0 \\
Net tracks $/ \mathrm{cm}^{2}$ & 86 & 204 & 275 & 429 & 637 & 96 & 288.5 & 386.5 & 507.5 & 643.5 \\
Tracks $/ \mathrm{cm}^{2} / \mathrm{mSv}$ & 86 & 102 & 91.6 & 85.9 & 63.7 & 96 & 144.25 & 128 & 101.5 & 64.3 \\
Avg. tracks $/ \mathrm{cm}^{2} / \mathrm{mSv}$ & \multicolumn{3}{c}{$91.4 \pm 7.56$} & & \multicolumn{4}{c}{$117.4 \pm 22.4$} \\
\hline
\end{tabular}

The response is relatively linear up to $5.0 \mathrm{mSv}$. For doses above $10 \mathrm{mSv}$, the tracks are overlapping due to very high track density. A good fraction of the tracks are rejected by the software of the counting system in which the range selection for track dia. is $70-200 \mu \mathrm{m}$, and hence the response does not fall in the linear region of the dose response curve.

\section{Conclusions}

Carbon Dioxide $\left(\mathrm{CO}_{2}\right)$ laser beam may be employed for cutting of CR-39 sheets used for personnel monitoring as the increase in background is within the statistical variation in background of normal CR-39 sheets and laser cutting of the sheets will ensure precision and save on the efforts involved in cutting large number of sheets manually.

The addition of a pre-etch step to normal processing of CR-39, provides a reduction in the background tracks by $40 \%$. The pre-etch step removes $\approx 1$ micron thickness from the surface. Superficial impurities such as adhesives from the polyethylene protective covering, alpha particle tracks and scratches are removed, leading to the reduction in the background tracks. The energy response and dose response of CR- 
39 for this process need to be evaluated before implementation for routine monitoring.

With the addition of chemical pre-etch step to normal processing by ETECE, The minimum measurable dose got reduced from $0.12 \mathrm{mSv}$ to $0.06 \mathrm{mSv}$ for manual cut CR-39 and from $0.3 \mathrm{mSv}$ to $0.09 \mathrm{mSv}$ for laser cut foils. For personnel monitoring purposes where there are large numbers of foils to be processed, it is valuable to reduce the total processing time from $6 \mathrm{hrs} 40 \mathrm{~min}$ for processing by ETECE to $5 \mathrm{hrs}$ 40 min by introducing pre-etch step, leading to more flexibility in processing the foils within the working hours.

\section{References}

[1] R J Tanner, D T Bartlett and L G Hager Radiation Measurements 40549 (2005)

[2] J R Harvey and A R Weeks Radiation Protection Dosimetry 2089 (1987)

[3] M P Dhairyawan, P K Marathe and O P Massand Radiation Measurements 36435 (2003)

[4] Dale E Hankins, Steven G Homann and Brooke Buddemeier Personnel Neutron Dosimetry Using Electrochemically Etched CR-39 foils Publication by Lawrence Livermore National Laboratory (1989)

[5] Y V Rao, S Kar, G Valli, D G Joshi, V K Chaddaa, R K Nigam, OP Massand, M P Dhairyawan and P K Marathe Image Analysis System for Automation in Fast Neutron Personnel Monitoring using CR-39 Solid State Track Detector, International Conference on Automation ICAUTO-95 (Indore, India) (1995) 